\title{
BANACH SPACES OF CONTINUOUS VECTOR-VALUED FUNCTIONS OF ORDINALS
}

\author{
ELÓI MEDINA GALEGO \\ Department of Mathematics, IME, University of São Paulo, \\ São Paulo 05315-970, Brazil (eloi@ime.usp.br)
}

(Received 5 July 1998)

\begin{abstract}
Let $X$ be a Banach space and $\xi$ an ordinal number. We study some isomorphic classifications of the Banach spaces $X^{\xi}$ of the continuous $X$-valued functions defined in the interval of ordinals $[1, \xi]$ and equipped with the supremum norm. More precisely, first we use the continuum hypothesis to give an isomorphic classification of $C(I)^{\xi}, \xi \geqslant \omega_{1}$. Then we present a characterization of the separable Banach spaces $X$ that are isomorphic to $X^{\xi}, \forall \xi, \omega \leqslant \xi<\omega_{1}$. Finally, we show that the isomorphic classifications of $\left(C(I) \oplus F^{*}\right)^{\xi}$ and $\ell_{\infty}(\mathbb{N})^{\xi}$, where $F$ is the space of Figiel and $\omega \leqslant \xi<\omega_{1}$ are similar to that of $\mathbb{R}^{\xi}$ given by Bessaga and Pelczynski.
\end{abstract}

Keywords: continuous vector-valued functions; isomorphic classifications of Banach spaces

AMS 2000 Mathematics subject classification: Primary 46B03; 46B20; 46E15

\section{Introduction}

As in [1], $X$ being a Banach space and $\xi$ an ordinal number, $X^{\xi}$ will indicate the Banach space of the continuous $X$-valued functions defined in the interval of ordinals $[1, \xi]$ and equipped with the supremum norm. $C(I)$ being the Banach space of continuous functions defined in the interval $I=[0,1]$ of the real line $\mathbb{R}$ with the supremum norm, it follows from Milutin's Theorem (see $[\mathbf{1 8}$, p. 379]) that

$$
C(I)^{\xi} \text { is isomorphic to } C(I), \quad \forall \xi, \quad \omega \leqslant \xi<\omega_{1} .
$$

Initially, using the continuum hypothesis we give an isomorphic classification of the Banach spaces $C(I)^{\xi}, \xi \geqslant \omega_{1}$ (Theorem 3.1). Afterwards, inspired by Bourgain [15] we exhibit a characterization of the separable Banach spaces $X$ such that $X^{\xi}, \forall \xi, \omega \leqslant \xi<\omega_{1}$ is isomorphic to $X$ (Theorem 4.1). Next, by Pisier's Theorem, we will generalize a result from Samuel [16], and we show that the presence of $F^{*}$, where $F$ is the space of Figiel [6], together with $C(I)$ annihilates (1.1), since the isomorphic classification of $\left(C(I) \oplus F^{*}\right)^{\xi}$, $\omega \leqslant \xi<\omega_{1}$ is similar to that of $\mathbb{R}^{\xi}$ given in [1] (Corollary 5.6). Finally, we will prove that the same happens with the isomorphic classification of $\ell_{\infty}(\mathbb{N})^{\xi}, \omega \leqslant \xi<\omega_{1}$, where $\ell_{\infty}(\mathbb{N})$ is the Banach space of the bounded sequences with the supremum norm (Corollary 5.8). These results motivated the definition of $\omega_{1}$ cancellable Banach space (Definition 5.9).

\section{Preliminaries}

To fix the notation, let us recall some definitions. If $X$ and $Y$ are Banach spaces, then $Y$ is isomorphic to a closed subspace of $X, Y \hookrightarrow X$, if there is a one-to-one bounded linear 
operator from $Y$ into $X ; Y$ is said to be isomorphic to $X, X \sim Y$, provided there is a one-to-one bounded linear operator from $X$ onto $Y$, and $Y$ is a quotient of $X, X \rightarrow Y$, if there is a surjective bounded linear operator from $X$ onto $Y$. The notation $\hat{\otimes}_{n} X$ will indicate the injective tensor product of $n$ isomorphic copies of $X, n<\omega$.

Let $\Gamma$ be a set. By $C_{0}(\Gamma, X)$ we denote the Banach space of $X$-valued functions defined on $\Gamma$ such that for any positive $\varepsilon$ the set $\{\gamma \in \Gamma:\|f(\gamma)\| \geqslant \varepsilon\}$ is finite, with the supremum norm, and by $\ell_{1}(\Gamma, X)$ we denote the Banach space of all absolutely summable $X$-functions defined on $\Gamma$.

If $\alpha$ is an ordinal number and $X$ is a Banach space, we set $X_{0}^{\alpha}=\left\{f \in X^{\alpha}: f(\alpha)=0\right\}$. The cardinality of the ordinal number $\xi$ will be denoted by $\bar{\xi}$. The notation $\omega_{1}$ will denote the first non-denumerable ordinal. If $\alpha$ is a non-denumerable regular ordinal and $\gamma$ is any ordinal, we will denote by $\wedge_{\gamma}^{\alpha}$ the subset of $[1, \gamma]$ consisting of limit ordinals that are not limits of sets of cardinality strictly smaller than $\bar{\alpha}$.

The density character dens $X$ of a Banach space $X$ is the smallest cardinal number $\delta$ such that there exists a set of cardinality $\delta$ dense in $X$.

Let $\gamma$ be an ordinal. A $\gamma$-sequence in a set $A$ is the image of a function $f:[1, \gamma[\rightarrow A$ and will be denoted by $\left(x_{\theta}\right)_{\theta<\gamma}$. If $A$ is a topological space and $\beta$ is an ordinal, we will say that the $\gamma$-sequence is $\beta$-continuous if, for every $\beta$-sequence of ordinals $\left(\theta_{\xi}\right)_{\xi<\beta}$ on $[1, \gamma]$ that converges to $\theta_{\beta}$ when $\xi$ converges to $\beta$, we have that $x_{\theta_{\xi}}$ converges to $x_{\theta_{\beta}}$.

Let $X$ be a Banach space. By $X_{s}$ we will denote the set of $F \in X^{* *}$ having the following property: for every $\omega$-sequence $\left(x_{n}^{*}\right)_{n<\omega}$ in $X^{*}$ such that $x_{n}^{*}(x) \stackrel{n \rightarrow \omega}{\longrightarrow} 0$, for all $x \in X$, we have $F\left(x_{n}^{*}\right) \stackrel{n \rightarrow \omega}{\longrightarrow} 0 . X$ is said to have Mazur's property (also, $d$-complete [9] or $\mu B$-spaces [19]) if $X_{s}=c X$, where $c X$ is the canonical image of $X$ in $X^{* *}$. The class of Banach spaces with Mazur's property includes the weakly compactly generated (WCG) Banach spaces and, therefore, the separable Banach spaces. See [11] for more examples of Banach spaces having this property.

Let $\alpha$ be a non-denumerable regular ordinal, $\varphi$ any ordinal, and $X$ a Banach space. By $X_{\alpha}^{\varphi}$ we will denote the set of $F \in X^{* *}$ having the following property: for every limit ordinal $\beta<\alpha$ and for every $\varphi$-sequence $x^{\eta}=\left(x_{\xi}^{*}(\eta)\right)_{\xi<\beta}$ of $\beta$-sequences of $X^{*}$ such that there exists $K \in \mathbb{R}$ with $\left\|x_{\xi}^{*}(\eta)\right\| \leqslant K, \forall \eta<\varphi, \forall \xi<\beta$ and such that $x_{\xi}^{*}(\eta)(x) \stackrel{\xi \rightarrow \beta}{\longrightarrow} 0$, $\forall x \in X$, uniformly in $\eta$, we have $F\left(x_{\xi}^{*}(\eta)\right) \stackrel{\xi \rightarrow \beta}{\longrightarrow} 0$ uniformly in $\eta$.

We say that the Banach spaces $X$ and $Y$ are totally incomparable if $X$ and $Y$ have no isomorphic closed subspaces of infinite dimension.

If $T: X \rightarrow Y$ is a surjective bounded linear operator and $B_{X}$ and $B_{Y}$ are the closed unit balls of $X$ and $Y$ respectively, we define $r_{0}(T)=\inf \left\{r>0: B_{Y} \subset r T\left(B_{X}\right)\right\}$.

Other notations are standard in conformity with $[\mathbf{1 8}]$.

\section{Isomorphic classification of the Banach spaces $C(I)^{\xi}, \xi \geqslant \omega_{1}$}

Our main aim here is to prove the following theorem, which provides the isomorphic classification of the Banach spaces $C(I)^{\xi}, \xi \geqslant \omega_{1}$, and for that we will suppose the continuum hypothesis, that is $2^{\aleph_{0}}=\aleph_{1}$. 
Theorem 3.1. Let $\alpha$ be an initial non-denumerable ordinal and $X$ a separable Banach space with $X^{\omega} \sim X$ and dens $X^{*}=2^{\aleph_{0}}$.

(1) If $\alpha$ is singular, then $X^{\xi} \sim X^{\eta}$ with $\xi \leqslant \eta$ and $\bar{\xi}=\bar{\eta}=\bar{\alpha}$ if and only if $\eta<\xi^{\omega}$.

(2) If $\alpha$ is regular, then
(a) $X^{\alpha} \sim X^{\alpha \eta}$, with $1 \leqslant \eta \leqslant \omega_{1}$ if and only if $\eta<\omega_{1}$;
(b) $X^{\alpha \xi} \sim X^{\alpha \eta}$, with $\omega_{1} \leqslant \xi \leqslant \eta \leqslant \alpha$ if and only if $\bar{\xi}=\bar{\eta}$;
(c) $X^{\xi} \sim X^{\eta}, \quad$ with $\alpha^{2} \leqslant \xi \leqslant \eta$ and $\bar{\xi}=\bar{\eta}=\bar{\alpha}$ if and only if $\eta<\xi^{w}$.

We will need some auxiliary results.

Lemma 3.2. Let $X$ be a Banach space having Mazur's property and $\gamma$ be any ordinal, then

$$
\frac{\left(X^{\gamma}\right)_{\omega_{1}}^{\omega}}{c X^{\gamma}} \sim C_{0}\left(\wedge_{\gamma}^{\omega_{1}}, X\right)
$$

Proof. This is similar to the proof of Corollary 2.8 in [7], only noticing that the Statement (b) of the proof of Proposition 2b is also true in this case, since, if $F \in$ $\left(X^{\gamma}\right)_{\omega_{1}}^{\omega}$ and $H$ is the canonical isomorphism from $\ell_{1}\left([1, \gamma], X^{*}\right)$ onto $\left(X^{\gamma}\right)^{*}$, then $H^{*}(F)=$ $\left(F_{\theta}\right)_{\theta<\gamma+1}$ is $\beta$-continuous, $\forall \beta, \beta<\omega_{1}$. Indeed, let $\beta<\omega_{1}$ and $\left(\theta_{\xi}\right)_{\xi<\beta}$ be a $\beta$-sequence of ordinals in $[1, \gamma]$ converging to $\theta_{\beta}$ when $\xi$ converges to $\beta$.

Now suppose that $\left(F_{\theta_{\xi}}\right)_{\xi<\beta}$ does not converge to $F_{\theta_{\xi}}$ when $\xi$ converges to $\beta$. So there is $\varepsilon>0$ and a strictly increasing $\omega$-sequence of ordinals $\left(\xi_{n}\right)_{n<\omega}$ converging to $\beta$ and a $\omega$ sequence $\left(x_{n}^{*}\right)_{n<\omega}$ of elements of the unit ball of $X^{*}$ such that $\left\|F_{\theta_{\xi_{n}}}\left(x_{n}^{*}\right)-F_{\theta_{\beta}}\left(x_{n}^{*}\right)\right\| \geqslant \varepsilon$. Let $P_{\xi_{m}}^{n}$ be in $\ell_{1}\left([1, \gamma], X^{*}\right)$ defined by $P_{\xi_{m}}^{n}(\theta)=x_{n}^{*}$ if $\theta=\theta_{\xi_{m}}$ and $P_{\xi_{m}}^{n}(\theta)=0$ if $\theta=\theta_{\xi_{m}}, \forall n, m<\omega$, so $H P_{\xi_{m}}^{n}(f)=x_{n}^{*} f\left(\theta_{\xi_{m}}\right)$ and, therefore, $\left|H\left(P_{\theta_{\xi_{m}}}^{n}-P_{\theta_{\beta}}^{n}\right)(f)\right| \leqslant$ $\left\|x_{n}^{*}\right\|\left\|f\left(\theta_{\xi_{m}}\right)-f\left(\theta_{\beta}\right)\right\| \stackrel{m \rightarrow \omega}{\longrightarrow} 0, \forall f$, uniformly in $n$ and $\left\|H\left(P_{\theta_{\xi_{m}}}^{n}-P_{\theta_{\beta}}^{n}\right)\right\| \leqslant 2\|H\|, \forall n$, $m<\omega$. Thus, $F H\left(P_{\theta_{\xi_{m}}}^{n}-P_{\theta_{\beta}}^{n}\right)=F_{\theta_{\xi_{m}}}\left(x_{n}^{*}\right)-F_{\theta_{\beta}}\left(x_{n}^{*} \stackrel{m \rightarrow \omega}{\longrightarrow} 0\right.$ uniformly in $n$, which is absurd.

Now, we remark that the argument presented in the proof of Lemma 2 in $[\mathbf{1}]$ also proves the following result.

Lemma 3.3. Let $\xi$ be a limit ordinal and $X$ a Banach space. If, for every $\beta<\xi$, $\mathbb{R}^{\xi} \nrightarrow X^{\beta}$ holds, then $\mathbb{R}^{\xi^{\omega}} \nrightarrow X^{\xi}$.

Lemma 3.4. Let $\xi$ be a non-denumerable ordinal and $X$ a separable Banach space, then $\mathbb{R}^{\xi^{\omega}} \nrightarrow X^{\xi}$.

Proof. Let us suppose that (a) $\mathbb{R}^{\xi^{\omega}} \hookrightarrow X^{\xi}$, thus $\mathbb{R}^{\xi} \hookrightarrow \mathbb{R}^{\xi^{\omega}} \hookrightarrow X^{\xi}$, so we can consider $\xi_{0}=\min \left\{\theta: \exists m, m<\omega, \mathbb{R}^{\xi} \hookrightarrow\left(\hat{\otimes}_{m} X\right)^{\theta}\right\}$. Let $m_{0}, m_{0}<\omega$, be such that (b) $\mathbb{R}^{\xi} \hookrightarrow\left(\hat{\otimes}_{m_{0}} X\right)^{\xi_{0}}$. It suffices to show that $\xi_{0}$ is finite to come to a contradiction, because, in this case, $\left(\hat{\otimes}_{m_{0}} X\right)^{\xi_{0}}$ is separable and $\mathbb{R}^{\xi}$ is not. 
We suppose that $\xi_{0}$ is infinite and we note that (c) $\mathbb{R}^{\xi_{0}} \hookrightarrow\left(_{\mathrm{\otimes}_{m_{0}+1}} X\right)^{\beta}, \forall \beta, \beta<\xi_{0}$. Indeed, otherwise there exists $\beta_{1}, \beta_{1}<\xi_{0}$ such that by using item (b), Theorem 20.5.6 in $[\mathbf{1 8}]$ and Proposition 7 in $[\mathbf{4}$, p. 225], we have

$$
\begin{aligned}
\mathbb{R}^{\xi} \hookrightarrow\left(\hat{\otimes}_{m_{0}} X\right)^{\xi_{0}} & =\mathbb{R}^{\xi_{0}} \hat{\hat{\otimes}}\left(\hat{\hat{\otimes}}_{m_{0}} X\right) \hookrightarrow\left(\hat{\hat{\otimes}}_{m_{0}+1} X\right)^{\beta_{1}} \hat{\hat{\otimes}}\left(\hat{\otimes}_{m_{0}} X\right) \\
& =\mathbb{R}^{\beta_{1}} \hat{\hat{\otimes}}\left(\hat{\otimes}_{m_{0}+1} X\right) \hat{\hat{\otimes}}_{m_{0}} X=\left(\hat{\hat{\otimes}}_{2 m_{0}+1} X\right)^{\beta_{1}},
\end{aligned}
$$

which is absurd because of the choice of $\xi_{0}$. We state that $\xi_{0}$ is a limit ordinal. Indeed, if $\xi_{0}=\xi_{1}+n$, for some $n, 0 \leqslant n<\omega$ and $\xi_{1}$ infinite, then $n+\xi_{1}=\xi_{1}$ and, from Property II in [1, p. 54], it follows that $\left(\hat{\otimes}_{m_{0}} X\right)^{\xi_{0}} \sim\left(\hat{\otimes}_{m_{0}} X\right)^{\xi_{1}}$, so, by the minimality of $\xi_{0}$, we conclude that $n=0$.

We can use Lemma 3.3 and item (c) to conclude that (d) $\mathbb{R}^{\xi_{0}^{w}} \nrightarrow\left(\hat{\otimes}_{m_{0}+1} X\right)^{\xi_{0}}$. Since $\xi_{0} \leqslant \xi$, and bearing (a) and (b) in mind, we have

$$
\mathbb{R}^{\xi_{0}^{\omega}} \hookrightarrow \mathbb{R}^{\xi^{\omega}} \hookrightarrow X^{\xi}=\mathbb{R}^{\xi} \hat{\otimes} X \hookrightarrow\left(\hat{\otimes}_{m_{0}} X\right)^{\xi_{0}} \hat{\otimes} X=\mathbb{R}^{\xi_{0}} \hat{\hat{\otimes}}\left(\hat{\otimes}_{m_{0}} X\right) \otimes X=\left(\otimes_{m_{0}+1} X\right)^{\xi_{0}},
$$

which is absurd because of $(d)$.

So $\xi_{0}$ must be finite.

Proof of Theorem 3.1.

(1) Let $\alpha$ be singular. If $X^{\xi} \sim X^{\eta}$ with $\xi \leqslant \eta$ and we also suppose $\xi^{\omega} \leqslant \eta$, then

$$
\mathbb{R}^{\xi^{\omega}} \hookrightarrow \mathbb{R}^{\eta} \hookrightarrow X^{\eta} \sim X^{\xi}
$$

which is absurd by Lemma 3.4.

Conversely, if $\xi \leqslant \eta, \bar{\xi}=\bar{\eta}=\bar{\alpha}$ and $\eta<\xi^{\omega}$, then from Theorem 1 in [10] follows that $\mathbb{R}^{\xi} \sim \mathbb{R}^{\eta}$, so $\mathbb{R}^{\xi \hat{\otimes}} X \sim \mathbb{R}^{\eta} \hat{\hat{\otimes}} X$, that is, $X^{\xi} \sim X^{\eta}$.

(2) Let $\alpha$ be regular.

(a) If $X^{\alpha} \sim X^{\alpha \eta}$ with $1 \leqslant \eta \leqslant \omega_{1}$, then we consider two cases. If $\alpha=\omega_{1}$, then, from Remark 2.3 in [7], we have

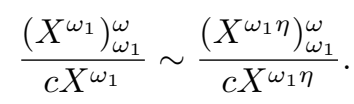

Then, by Lemma 3.2, $C_{0}\left(\wedge_{\omega_{1}}^{\omega_{1}}, X\right) \sim C_{0}\left(\wedge_{\omega_{1} \eta}^{\omega_{1}}, X\right)$, that is $X \sim C_{0}(\Gamma, X)$, where $\Gamma=$ $[1, \eta]$ (see $[\mathbf{1 0}]$ ). Since $X$ is separable, we conclude that $\eta<\omega_{1}$.

If $\alpha>\omega_{1}$, then again from Remark 2.3 in [7] it follows that

$$
\frac{\left(X^{\alpha}\right)_{\alpha}^{\omega_{1}}}{c X^{\alpha}} \sim \frac{\left(X^{\alpha \eta}\right)_{\alpha}^{\omega_{1}}}{c X^{\alpha \eta}}
$$

Since we have the hypothesis that $\operatorname{dim} X^{*}=2^{\aleph_{0}}=\aleph_{1}<\bar{\alpha}$, we can apply Corollary 2.8 of [7] to obtain $C_{0}\left(\wedge_{\alpha}^{\alpha}, X\right) \sim C_{0}\left(\wedge_{\alpha \eta}^{\alpha}, X\right)$, that is $X \sim C_{0}(\Gamma, X)$, where $\Gamma=[1, \eta]$; the separability of $X$ implies $\eta<\omega_{1}$.

Conversely, let $\eta$ be $1 \leqslant \eta<\omega_{1}$. It suffices to prove that

$$
X^{\alpha} \sim X^{\alpha \theta} \quad \forall \theta, \omega \leqslant \theta<\omega_{1} .
$$


Indeed, if $1 \leqslant n<\omega$, from $X^{\alpha} \sim X^{\alpha \omega}$, Property III in [1, p. 54] and Theorem 2 in $[\mathbf{1 0}]$, it follows that

$$
X^{\alpha n} \sim\left(X^{\alpha}\right)^{n} \sim\left(X^{\alpha \omega}\right)^{n} \sim X^{(\alpha \omega) n} \sim X^{\alpha(\omega n)} \sim \mathbb{R}^{\alpha(\omega n)} \hat{\hat{\theta}} X \sim \mathbb{R}^{\alpha \omega} \hat{\hat{\otimes}} X \sim X^{\alpha} .
$$

To see (3.1), firstly we note that

$$
\mathbb{R}^{\alpha \omega} \sim \mathbb{R}_{0}^{\alpha \omega} \sim\left(\mathbb{R}_{0}^{\alpha}\right)_{0}^{\omega} \sim\left(\mathbb{R}^{\alpha}\right)^{\omega} \sim \mathbb{R}^{\alpha} \hat{\otimes} \mathbb{R}^{\omega}
$$

The first and the third isomorphisms are Remark 2.1 in $[\mathbf{1}$, p. 55], the second isomorphism follows from Corollary 3.1 in $[\mathbf{1 0}]$, and the fourth isomorphism follows from Corollary 7.7.6 and Theorem 20.5.6 in [18].

Finally, let $\theta$ be $\omega \leqslant \theta<\omega_{1}$, thus

$$
\begin{aligned}
X^{\alpha \theta} \sim \mathbb{R}^{\alpha \theta} \hat{\otimes} X \sim \mathbb{R}^{\alpha \omega} \hat{\otimes} X \sim\left(\mathbb{R}^{\alpha} \hat{\otimes} \mathbb{R}^{\omega}\right) \hat{\otimes} X \\
\\
\sim \mathbb{R}^{\alpha} \hat{\otimes}\left(\mathbb{R}^{\omega} \hat{\otimes} X\right) \sim \mathbb{R}^{\alpha} \hat{\otimes} X^{\omega} \sim \mathbb{R}^{\alpha} \hat{\otimes} X \sim X^{\alpha} .
\end{aligned}
$$

The second isomorphism follows from Theorem 2 in [10].

(b) If $X^{\alpha \xi} \sim X^{\alpha \eta}$ with $\omega_{1} \leqslant \xi \leqslant \eta \leqslant \alpha$, we can suppose $\alpha>\omega_{1}$, because, if $\alpha=\omega_{1}$, then $\xi=\eta=\omega_{1}$ and we have nothing to prove. So as in the proof of the second case in (a) we obtain that $C_{0}\left(\wedge_{\alpha \xi}^{\alpha}, X\right) \sim C_{0}\left(\wedge_{\alpha \eta}^{\alpha}, X\right)$, that is $C_{0}\left(\Gamma_{1}, X\right) \sim C_{0}\left(\Gamma_{2}, X\right)$, where $\Gamma_{1}=[1, \xi]$ and $\Gamma_{2}=[1, \eta]$, thus, the separability of $X$ implies $\bar{\xi}=\bar{\eta}$.

Conversely, if $\omega_{1} \leqslant \xi \leqslant \eta \leqslant \alpha$ with $\bar{\xi}=\bar{\eta}$, then Theorem 2 in [10] implies that $\mathbb{R}^{\alpha \xi} \sim \mathbb{R}^{\alpha \eta}$, so $X \hat{\otimes} \mathbb{R}^{\alpha \xi} \sim X \hat{\otimes} \mathbb{R}^{\alpha \eta}$.

(c) If $X^{\xi} \sim X^{\eta}$ with $\xi \leqslant \eta$, then, as it was proved in the case in which $\alpha$ is singular, we have $\eta<\xi^{\omega}$. Conversely, if $\alpha^{2} \leqslant \xi \leqslant \eta, \bar{\xi}=\bar{\eta}=\bar{\alpha}$, then Theorem 2 in [10] implies that $\mathbb{R}^{\xi} \sim \mathbb{R}^{\eta}$, so $X \hat{\otimes} \mathbb{R}^{\xi} \sim X \hat{\otimes} \mathbb{R}^{\eta}$.

Question 3.5. Give an isomorphic classification of the Banach spaces $C(I)^{\xi}, \xi \geqslant \omega_{1}$, without using the continuum hypothesis.

Remark 3.6. For each $\gamma, 1 \leqslant \gamma<\omega_{\xi+1}$, where $\omega_{\xi+1}$ is the first ordinal of cardinality $\aleph_{\xi+1}$, we define $K_{\gamma}=\left[1, \omega_{\xi}^{\omega^{\gamma}}\right] \times I, \omega_{\xi}$ being the first ordinal of cardinality $\aleph_{\xi}$. It follows from Lemma 3.4 that $C\left(K_{\eta_{1}}\right) \hookrightarrow C\left(K_{\xi_{1}}\right)$, for every $1 \leqslant \xi_{1}<\eta_{1}<\omega_{\xi+1}$. then

Indeed, let $\theta_{\xi_{1}}=\omega_{\xi}^{\omega^{\xi_{1}}}$ and $\theta_{\gamma_{1}}=\omega_{\xi}^{\omega^{\gamma_{1}}}$, thus $\theta_{\xi_{1}}^{\omega}=\omega_{\xi}^{\omega^{\xi_{1}+1}} \leqslant \theta_{\gamma_{1}}$. If $C\left(K_{\eta_{1}}\right) \hookrightarrow C\left(K_{\xi_{1}}\right)$,

$$
\mathbb{R}_{\xi_{\xi_{1}}}^{\omega_{1}} \hookrightarrow \mathbb{R}^{\theta_{\gamma_{1}}} \hookrightarrow C\left(K_{\eta_{1}}\right) \hookrightarrow C\left(K_{\xi_{1}}\right)=C(I)^{\theta_{\xi_{1}}}
$$

which is a contradiction.

So, for each $\aleph_{\xi} \geqslant 2^{\aleph_{0}}$, there exists at least $\aleph_{\xi+1}$ perfect compacts $K$ of the cardinality $\aleph_{\xi}$, such that $C(K)$ are isomorphically different.

Question 3.7. Under the continuum hypothesis, are there more than $\aleph_{2}$ perfect compacts $K$ of cardinality $2^{\aleph_{0}}$, such that $C(K)$ are isomorphically different? 
4. Characterization of the separable Banach spaces satisfying $X^{\xi} \sim X, \forall \xi$, $\omega \leqslant \xi<\omega_{1}$

If $X$ is a Banach space and $K$ a compact, $C(K, X)$ will indicate the Banach space of the continuous $X$-valued functions defined on $K$ and equipped with the supremum norm.

It follows from the Milutin's Theorem that if $X$ is isomorphic to $C(I)$, then $X$ satisfies the following equation: $X^{\xi} \sim X, \forall \xi, \omega \leqslant \xi<\omega_{1}$. In this section we will prove Theorem 4.1, which gives an isomorphic characterization of the separable Banach spaces satisfying such an equation.

Theorem 4.1. Let $X$ be a separable Banach space. $X$ satisfies the equation $X^{\xi} \sim X$, $\forall \xi, \omega \leqslant \xi<\omega_{1}$, if and only if $C(I, X) \sim X$.

Proof. If $X$ is a Banach space satisfying

$$
C(I, X) \sim X \quad \text { and } \quad \xi, \quad \omega \leqslant \xi<\omega_{1},
$$

then, from Lemma 21.5.1 of [18], we have $\mathbb{R}^{\xi} \hat{\otimes} C(I, X) \sim \mathbb{R}^{\xi} \hat{\otimes} X$. Now, from Theorem 20.5.6 of [18], we obtain $\mathbb{R}^{\xi} \hat{\otimes} C(I) \hat{\otimes} X \sim X^{\xi}$. So, from Milutin's Theorem, $C(I, X) \sim$ $X^{\xi}$. But, bearing in mind (4.1), we conclude that $X \sim X^{\xi}$.

The converse follows immediately from the following proposition.

Proposition 4.2. Let $X$ and $B$ be separable Banach spaces with $X^{\xi} \sim B, \forall \xi, \omega \leqslant$ $\xi<\omega_{1}$, then $C(I, X) \sim B$.

Proof. (Inspired by [15].) Let $2^{I}$ be the space of all compact subsets of $I$ endowed with the Hausdorff metric

$$
d(A, B)=\max \left\{\max _{a \in A} \operatorname{dist}(a, B), \max _{b \in B} \operatorname{dist}(b, A)\right\} .
$$

Let $Y=\left\{K \in 2^{I}: C(K, X) \sim B\right\}$. For each $n<\omega, Y_{n}=\left\{K \in 2^{I}: \exists \bar{T}: C(I, X) \rightarrow B\right.$ a bounded linear operator, $\|\bar{T}\| \leqslant 1$ and $\bar{L}: B \rightarrow C(I, X)$ a bounded linear operator satisfying $(1 / n)\left\|f_{\left.\right|_{K}}\right\| \leqslant\|\bar{T}(f)\| \leqslant\left\|f_{\left.\right|_{K}}\right\|, \forall f \in C(I, X)$ and $\bar{T} \bar{L}(b)=b, \forall b \in B,\|\bar{L}\| \leqslant$ $n\}$.

Firstly, we remark that $Y=\bigcup_{n<\omega} Y_{n}$. Indeed, supposing that $K \in Y$, there exists $T: C(K, X) \rightarrow B$, an isomorphism onto the image (we can suppose $\|T\| \leqslant 1$ ), and $L: B \rightarrow C(K, X)$, a bounded linear operator ( $L$ is the inverse of $T$ ), satisfying $T L(b)=b$, $\forall b \in B$.

Let $n<\omega$ be such that $\|L\| \leqslant n$. We define $\bar{T}: C(I, X) \rightarrow B$ by $\bar{T}(g)=T\left(g_{\left.\right|_{K}}\right)$ and $L: B \rightarrow C(I, K)$, by $\bar{L}(b)=E L(b)$, where $E$ is a linear extension operator (see $[\mathbf{1 8}$, p. 365]), so $\left\|g_{\left.\right|_{K}}\right\|=\left\|L T\left(g_{\left.\right|_{K}}\right)\right\| \leqslant n\left\|T\left(g_{\left.\right|_{K}}\right)\right\|$,

(I) $(1 / n)\left\|g_{\left.\right|_{K}}\right\| \leqslant\|\bar{T}(g)\|=\left\|T\left(g_{\left.\right|_{K}}\right)\right\| \leqslant\left\|g_{\left.\right|_{K}}\right\|, \forall g \in C(I, X)$, and

(II) $\bar{T} \bar{L}(b)=\bar{T} E L(b)=T\left(E L(b)_{\left.\right|_{K}}\right)=T L(b)=b, \forall b \in b$, that is $K \in Y_{n}$. 
Conversely, supposing $K \in Y_{n}$ for some $n<\omega$, we define $T: C(K, X) \rightarrow B$ by $T=\bar{T} E$, where $E$ is a linear extension operator and $L: B \rightarrow C(K, X)$ by $L=R \bar{L}$, where $R$ is an operator defined by $R(f)=f_{\left.\right|_{K}}, \forall f \in C(I, X)$. Let $f \in C(K, X)$ and let $b \in B$, then

(III) $(1 / n)\|f\|=(1 / n)\left\|E(f)_{\left.\right|_{K}}\right\| \leqslant\|T(f)\|=\|\bar{T} E(f)\| \leqslant\left\|E(f)_{\left.\right|_{K}}\right\|=\|f\|$, that is $T$ is an isomorphism onto the image.

(IV) $T L(b)=\bar{T} E R \bar{L}(b)$ and, since $\bar{L}(b)_{\left.\right|_{K}}=(E R \bar{L}(b))_{\left.\right|_{K}}$ and $\|\bar{T}(f)\| \leqslant\left\|f_{\left.\right|_{K}}\right\|, \forall f \in$ $C(I, K)$, it follows that $\bar{T}(E R \bar{L}(b))=\bar{T}(\bar{L}(b))=b$ and, therefore, $T L(b)=b$, $\forall b \in B$, so the image of $T$ is $B$, consequently $B \in Y$.

Next we will remark that each $Y_{n}$ is analytic. Let $A$ be the unit ball of $L(C(I, X), B)$ in the pointwise convergence topology, and let $J$ be the ball of radius $n$ of $L(B, C(I, X))$ also in the pointwise convergence topology.

We consider the Polish space $Z=2^{I} \times A \times J$ (see [3, p. 195]). Let $Q=\{(K, T, L) \in$ $Z:(1 / n)\left\|f_{\left.\right|_{K}}\right\| \leqslant\|T(f)\| \leqslant\left\|f_{\left.\right|_{K}}\right\|, \forall f \in C(I, X)$ and $\left.T L(b)=b, \forall b \in B\right\} . Q$ is a closed subset of $Z$, because, if $\left(K_{\gamma}, T_{\gamma}, L_{\gamma}\right)$ is a net of $Q$ converging to $(K, T, L)$ in $Z$, it follows that $(1 / n)\left\|f_{\left.\right|_{K}}\right\| \leqslant\|T(f)\| \leqslant\left\|f_{\left.\right|_{K}}\right\|, \forall f \in C(I, X)$, from the pointwise convergence of $T_{\gamma}$.

Next we show that $T L(b)=b, \forall b \in B$.

Let $b \in B$, from $T_{\gamma} \rightarrow T$, it follows that

(1) $T_{\gamma} L(b) \rightarrow T L(b)$,

and, from $L_{\gamma} \rightarrow L$, it follows that $L_{\gamma}(b) \rightarrow L(b)$ and, therefore,

(2) $T L_{\gamma}(b) \rightarrow T L(b)$.

Now, using

$$
\left\|T_{\gamma} L_{\gamma}(b)-T L_{\gamma}(b)\right\| \leqslant\left\|T_{\gamma}\right\|\left\|L_{\gamma}(b)-L(b)\right\|+\|T\|\left\|L_{\gamma}(b)-L(b)\right\|+\left\|T_{\gamma} L(b)-T L(b)\right\|
$$

with (1) and (2), we conclude that $b=T_{\gamma} L_{\gamma}(b) \rightarrow T L(b)$, that is $T L(b)=b$. So $(K, T, L) \in Q$ and, therefore, $Q$ is closed, consequently (see [3, p. 195]) $Q$ is a Polish space. Since $Y_{n}$ is a projection of $Q$ onto the $2^{I}$ axis, $Y_{n}$ is analytic and, therefore, so is $Y$ (see [3, p. 195]).

Let $D=\left\{K \in 2^{I}: K\right.$ is countable $\}$, by the hypothesis of the proposition, it follows that $D \subset Y$ (see [18, p. 155]), and, since $D$ is non-analytic (see [8]), there must be a non-denumerable compact subset $K$ of $I$, such that $C(K, X) \sim B$, and, from Milutin's Theorem and Corollary 21.5.2 of [18], it follows that $C(I, X) \sim B$.

Remark 4.3. Let $Y$ be a Banach space. It follows from Milutin's Theorem and from properties of injective tensor products that $X=C(I, Y)$ satisfies

$$
C(I, X) \sim X
$$


It will be shown (Corollary 5.6) that there exists a separable Banach space $W, C(I) \hookrightarrow$ $W$, such that $X=C(I) \oplus W$ does not satisfy (4.2). (To see this, bear in mind that if $X$ satisfies (4.2), then $X^{\xi} \sim X^{\eta}, \forall \xi, \eta, \omega \leqslant \xi \leqslant \eta<\omega_{1}$.)

Now, since $C(I) \oplus C(I, Y) \sim C(I, Y)$, we have that $X=C(I) \oplus Y$ satisfies (4.2) if and only if

$$
C(I, Y) \sim C(I) \oplus Y .
$$

If $Y$ is isomorphic to a complemented subspace of $C(I)$ and $C(I) \nrightarrow Y$, then $Y$ satisfies (4.3). Indeed, from Corollary 1 of [12], we have $C(I) \oplus Y \sim C(I)$ and, therefore, $C(I, C(I) \oplus Y) \sim C(I, C(I))$, that is $C(I, Y) \sim C(I)$.

Question 4.4. Let $Y$ be a separable Banach space, $C(I) \nrightarrow Y$, such that $C(I, Y) \sim$ $C(I) \oplus Y$. Is it true that $Y$ is isomorphic to a complemented subspace of $C(I)$ ?

Question 4.5. Give a Banach space $X$ such that $X^{\omega_{1}} \sim X$.

\section{5. $\omega_{1}$ cancellable Banach spaces}

Next we present two Banach spaces $X$ containing subspaces isomorphic to $C(I)$ such that the isomorphic classifications of $X^{\xi}, \omega \leqslant \xi<\omega_{1}$ are similar to that of $\mathbb{R}^{\xi}$ given by Bessaga and Pelczynski in [1]. The first space is $C(I) \oplus F^{*}$, where $F$ is the Banach space considered by Figiel in [6] (Corollary 5.6), and the second is $\ell_{\infty}(\mathbb{N})$ (Corollary 5.8).

These results will be consequences of Theorem 5.1, and, in order to prove it, we will need some auxiliary results.

Theorem 5.1. Let $X^{*}$ and $F$ be totally incomparable Banach spaces satisfying $X \rightarrow$ $X^{\xi}, \forall \xi, \omega \leqslant \xi<\omega_{1}, F^{*}$ uniformly convex, $F^{n+1} \not F^{n}, \forall n, n<\omega$. If $\left(X \oplus F^{*}\right)^{\xi} \rightarrow$ $\left(X \oplus F^{*}\right)^{\eta}$, with $\omega \leqslant \xi \leqslant \eta<\omega_{1}$, then $\eta<\xi^{\omega}$.

Lemma 5.2. Let $X, Y$ and $Z$ Banach spaces, $T: X \oplus Y \rightarrow Z$ is a bounded linear operator such that $i_{1}^{*} T^{*}: Z^{*} \rightarrow X^{*}$ is an isomorphism onto the image, where $i_{1}$ is the canonical inclusion from $X$ in $X \oplus Y$. Then $T_{1}: X \rightarrow Z$ defined by $T_{1}(x)=T(x, 0)$, $\forall x \in X$ is onto $Z$.

Proof. From $T_{1}^{*}\left(z^{*}\right)(x)=z^{*}\left(T_{1}(x)\right)=z^{*}\left(T\left(i_{1}(x)\right)\right)=i_{1}^{*}\left(T^{*}\left(z^{*}(x)\right)\right), \forall z^{*} \in Z$, and $\forall x \in X$, it follows that $T_{1}^{*}$ is one-to-one and has a closed image. Then from Lemma 3 of $\left[\mathbf{5}\right.$, p. 488], we have that $T_{1}$ is onto $Z$.

Lemma 5.3. Let $\gamma$ be a denumerable ordinal, $X$ and $Y$ Banach spaces and $T: X_{0}^{\gamma} \oplus$ $Y \rightarrow Z$ a surjective bounded linear operator. Let $\beta<\gamma$ be such that $\left.T\right|_{X_{0}^{\beta} \oplus Y}: X_{0}^{\beta} \oplus Y \rightarrow$ $Z$ is not surjective and $r>r_{0}(T)$, then, for every $\varepsilon, 0<\varepsilon<1$, there exists $g \in X_{0}^{\gamma}$ with $g(\xi)=0, \forall \xi, \xi \leqslant \beta,\|g\| \leqslant r$ and $\|T(g)\| \geqslant \varepsilon$.

Proof. Let $\varepsilon$ be such that $0<\varepsilon<1$; choosing $\delta=1-\varepsilon$ and writing $X_{0}^{\gamma} \oplus Y=$ $X_{0}^{\beta} \oplus W \oplus Y$, where $W=\left\{f \in X_{0}^{\gamma}: f(\xi)=0, \forall \xi, \xi \leqslant \beta\right\}$ and indicating by $i_{1}$ the canonical inclusion from $X_{0}^{\beta} \oplus Y$ to $X_{0}^{\gamma} \oplus Y$, it follows from the previous lemma that 
there exists $z^{*} \in Z^{*},\left\|z^{*}\right\|=1$ such that $\left\|i_{1}^{*} T^{*} z^{*}\right\| \leqslant(\delta / 2 r)$, so, for every $f+y$ in $X_{0}^{\beta} \oplus Y$ with $\|f+y\| \leqslant r$, we have $\left\|z^{*} T(f+y)\right\| \leqslant \frac{1}{2} \delta$.

Let $z \in Z,\|z\|=1$ such that $\left\|z^{*}(z)\right\| \geqslant 1-\frac{1}{2} \delta$, since $T$ is surjective, then there exists $g^{1}+y_{1}$ in $X_{0}^{\gamma} \oplus Y,\left\|g^{1}+y_{1}\right\| \leqslant r$ such that $T\left(g^{1}+y_{1}\right)=z$. Let $g_{1}=g_{\left.\right|_{[\beta+1, \gamma]} ^{1}}^{1}$, thus:

$$
1-\frac{1}{2} \delta \leqslant\left\|z^{*} T\left(g^{1}+y_{1}\right)\right\| \leqslant\left\|z^{*} T\left(g^{1}-g_{1}+y_{1}\right)\right\|+\left\|z^{*} T\left(g_{1}\right)\right\| \leqslant \frac{1}{2} \delta+\left\|T\left(g_{1}\right)\right\|,
$$

so $\left\|T\left(g_{1}\right)\right\| \geqslant \varepsilon$.

Proposition 5.4. Let $X, Y$ and $Z$ be Banach spaces, $Y$ uniformly convex, $Z \rightarrow Y$ and $\alpha$ an infinite denumerable ordinal such that $\forall \beta, \beta<\alpha, Z^{\beta} \oplus X \not \nrightarrow Y^{\alpha}$. Then $Z^{\alpha} \oplus X \not \rightarrow Y^{\alpha^{\omega}}$.

Proof. (Inspired by [16].) $Y$ being uniformly convex, it follows from Pisier's Theorem (see $[\mathbf{1 4}$, p. 803]) that $Y$ admits an equivalent norm (that will be denoted by $\|\cdot\|$ ) and there exists $\delta>0$ and $p \in \mathbb{R}, 2<p<+\infty$ such that if $b \in \mathbb{R}_{+}$and $y_{1}, y_{2} \in Y$ with $\left\|y_{1}\right\| \geqslant 1$ and $\left\|y_{2}\right\| \geqslant \sqrt[p]{b}$, then either $\left\|y_{1}+y_{2}\right\| \geqslant \sqrt[p]{1+\delta b}$ or $\left\|y_{1}-y_{2}\right\| \geqslant \sqrt[p]{1+\delta b}$. So, given $y_{1}, y_{2}, \ldots, y_{n} \in Y$, with $\left\|y_{1}\right\|=1,\left\|y_{i}\right\| \geqslant \sqrt[p]{b}, i=2,3, \ldots, N$, there exists $c_{i} \in \mathbb{R}$, $\left|c_{i}\right|=1, i=1,2, \ldots, N$, such that $\left\|\sum_{i=1}^{N} c_{i} y_{i}\right\| \geqslant \sqrt[p]{1+(N-1) b \delta}$.

Let $\alpha=\omega^{\alpha_{1}} n_{1}+\omega^{\alpha_{2}} n_{2}+\cdots+\omega^{\alpha_{k}} n_{k}$ be in the Cantor normal form (see [18, p. 153]),

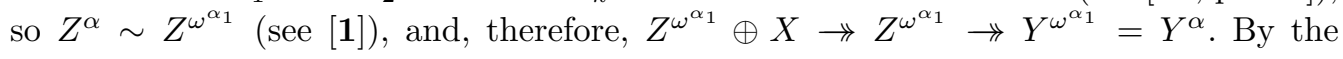
hypothesis of the proposition, we cannot have $\omega^{\alpha_{1}}<\alpha$, i.e. $\alpha=\omega^{\alpha_{1}}$, and so $\alpha$ is a prime component ordinal (see [18, p. 153]).

We also know that $\alpha^{\omega}=\omega^{\alpha_{1} \omega}$ and $Z^{\omega^{\alpha_{1}}} \sim Z_{0}^{\omega^{\alpha_{1}}}$ (see [1]). Then denying the thesis of the proposition, there will be $T$, a surjective bounded linear operator from $Z_{0}^{\omega^{\alpha_{1}}} \oplus X$ onto $Y^{\omega^{\alpha_{1} \omega}}$.

Let $r>r_{0}(T)$ and $\varepsilon, 0<\varepsilon<1$; we choose $s, s>0$ and $N, 1 \leqslant N<\omega$ such that $\varepsilon+s<1$ and $\|T\|(r+\varepsilon)<\sqrt[p]{1+(N-1) \varepsilon \delta}$. Let $y_{1} \in Y,\left\|y_{1}\right\|=1$ and $h \in Y^{\omega^{\alpha_{1} \omega}}$ defined by $h(\gamma)=y_{1}, \forall \gamma$. There exists $g^{1}, g^{1} \in Z_{0}^{\omega^{\alpha}} \oplus X$ such that $\left\|g^{1}\right\| \leqslant r$ and $T\left(g^{1}\right)=h_{1}$. Writing $g^{1}=g_{1}+f_{1}$, with $g_{1} \in Z_{0}^{\omega^{\alpha_{1}}}$ and $f_{1} \in X$, we get $\gamma_{1}, \gamma_{1}<\omega^{\alpha_{1}}$ such that $\left\|g_{1}(\gamma)\right\| \leqslant(\varepsilon / N), \forall \gamma \in\left[\gamma_{1}+1, \omega^{\alpha_{1}}\right]$.

Let us denote for every $\beta \in\left[0, \omega^{\alpha_{1}}\left[, \Delta_{\beta}^{1}=\left[\omega^{\alpha_{1}(N-1)} \beta+1, \omega^{\alpha_{1}(N-1)}(\beta+1)\right]\right.\right.$ and writing $W_{1}=\left\{f \in Y^{\omega^{\alpha_{1} N}}: \forall \beta, \beta \in\left[0, \omega^{\alpha_{1}}\left[, f\right.\right.\right.$ is constant in $\left.\Delta_{\beta}^{1}\right\}$. Let $P_{1}: Y^{\omega^{\alpha_{1} \omega}} \rightarrow W_{1}$ be the bounded linear operator defined by

$$
P_{1}(f)(\gamma)= \begin{cases}0, & \text { if } \gamma \in\left[\omega^{\alpha_{1} N}+1, \omega^{\alpha_{1} \omega}\right] \\ f(\gamma), & \text { if } \gamma=\omega^{\alpha_{1}(N-1)} \beta, \text { with } 1 \leqslant \beta \leqslant \omega^{\alpha_{1}}, \\ f\left(\omega^{\alpha_{1}(N-1)}(\beta+1)\right), & \text { if } \gamma=\omega^{\alpha_{1}(N-1)} \beta+\xi \\ & \text { with } 0 \leqslant \beta<\omega^{\alpha_{1}} \text { and } 1 \leqslant \xi<\omega^{\alpha_{1}(N-1)}\end{cases}
$$

$\forall f \in Y^{\omega^{\alpha_{1} \omega}}$.

Clearly, $W_{1}$ is a subspace of $Y^{\omega^{\alpha_{1} \omega}}$ isometric to $Y^{\omega^{\alpha_{1}}}$, and $P_{1} T$ is a surjective bounded linear operator from $Z_{0}^{\omega^{\alpha_{1}}} \oplus X$ to $W_{1}$ such that $r_{0}\left(P_{1} T\right) \leqslant r_{0}(T)$. 
The previous lemma applied to $\gamma_{1}$ and $\sqrt[p]{\varepsilon+s}$ implies that there exists $g_{2} \in Z_{0}^{\omega^{\alpha_{1}}}$, with $g_{2}(\xi)=0, \forall \xi, \xi \leqslant \gamma_{1},\left\|g_{2}\right\| \leqslant r$ and $\left\|P_{1} T\left(g_{2}\right)\right\| \leqslant \sqrt[p]{\varepsilon+s} ;$ so there exists $\beta_{1} \in\left[1, \omega^{\alpha_{1}}[\right.$ such that (1.1) $\left\|T g_{2}\left(\omega^{\alpha_{1}(N-1)} \beta_{1}\right)\right\| \geqslant \sqrt[p]{\varepsilon+s}$, and we can suppose that $\beta_{1}$ is not a limit ordinal satisfying $\left\|T g_{2}\left(\omega^{\alpha_{1}(N-1)} \beta_{1}\right)\right\| \geqslant \sqrt[p]{\varepsilon}$.

Let $\beta_{1}=\beta_{1}^{\prime}+1$. Since $T\left(g_{2}\right) \in Y^{\omega^{\alpha_{1} \omega}}$ we can find $\lambda_{1}, \lambda_{1} \in\left[0, \omega^{\alpha_{1}}[\right.$, such that for every $\gamma, \gamma \in\left[\omega^{\alpha_{1}(N-1)} \beta_{1}^{\prime}+\omega^{\alpha_{1}(N-2)} \lambda_{1}+1, \omega^{\alpha_{1}(N-1)} \beta_{1}\right]$, we have $\left\|T g_{2}(\gamma)\right\| \geqslant \sqrt[p]{\varepsilon}$. Since $g_{2} \in Z_{0}^{\omega^{\alpha_{1}}}$, there exists $\gamma_{2} \in\left[\gamma_{1}+1, \omega^{\alpha_{1}}\right]$ such that $\left\|g_{2}(\gamma)\right\| \leqslant(\varepsilon / N), \forall \gamma, \gamma \in\left[\gamma_{2}+1, \omega^{\alpha_{1}}\right]$. Let us denote for every $\beta, \beta \in\left[\lambda_{1}, \omega^{\alpha_{1}}\left[, \Delta_{\beta}^{2}=\left[\omega^{\alpha_{1}(N-1)} \beta_{1}^{\prime}+\omega^{\alpha_{1}(N-2)} \beta+1, \omega^{\alpha_{1}(N-1)} \beta_{1}^{\prime}+\right.\right.\right.$ $\left.\omega^{\alpha_{1}(N-2)}(\beta+1)\right]$, and, writing $W_{2}=\left\{f \in Y^{\omega^{\alpha_{1} \omega}}: \forall \beta, \beta \in\left[\lambda_{1}, \omega^{\alpha_{1}}\left[, f\right.\right.\right.$ is constant in $\Delta_{\beta}^{2}$, and $\left.\forall \gamma, \gamma \notin\left[\omega^{\alpha_{1}(N-1)} \beta_{1}^{\prime}+\omega^{\alpha_{1}(N-2)} \lambda_{1}+1, \omega^{\alpha_{1}(N-1)} \beta_{1}\right], f(\gamma)=0\right\}$. Let $P_{2}: Y^{\omega^{\alpha_{1} \omega}} \rightarrow W_{2}$ be the bounded linear operator defined by

$$
P_{2}(f)(\gamma)=\left\{\begin{aligned}
& 0, \text { if } \gamma \notin\left[\omega^{\alpha_{1}(N-1)} \beta_{1}^{\prime}+\omega^{\alpha_{1}(N-2)} \lambda_{1}+1, \omega^{\alpha_{1}(N-1)} \beta_{1}\right], \\
& f(\gamma), \text { if } \gamma=\omega^{\alpha_{1}(N-1)} \beta_{1}^{\prime}+\omega^{\alpha_{1}(N-2)} \beta, \text { with } \lambda_{1}+1 \leqslant \beta \leqslant \omega^{\alpha_{1}}, \\
& f\left(\omega^{\alpha_{1}(N-1)} \beta_{1}^{\prime}+\omega^{\alpha_{1}(N-2)}(\beta+1)\right), \\
& \text { if } \gamma=\omega^{\alpha_{1}(N-1)} \beta_{1}^{\prime}+\omega^{\alpha_{1}(N-2)} \beta+\xi, \\
& \text { with } \lambda_{1} \leqslant \beta<\omega^{\alpha_{1}} \text { and } 1 \leqslant \xi \leqslant \omega^{\alpha_{1}(N-2)},
\end{aligned}\right.
$$

$\forall f \in Y^{\omega^{\alpha_{1}} \omega}$ and $\forall \gamma, \gamma \in\left[1, \omega^{\alpha_{1} \omega}\right]$.

Since $\omega^{\alpha_{1}}$ is a prime component ordinal, it follows that $\left[\lambda_{1}, \omega^{\alpha_{1}}\right]$ is homeomorphic to $\left[1, \omega^{\alpha_{1}}\right]$ and, therefore, $W_{2}$ is a subspace of $Y^{\omega^{\alpha_{1} \omega}}$ isometric to $Y^{\omega^{\alpha_{1}}}$ and $P_{2} T$ is a

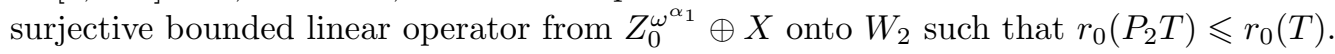

The previous lemma applied to $\gamma_{2}$ and $\sqrt[p]{\varepsilon+s}$ implies that there exists $g_{3} \in Z_{0}^{\omega^{\alpha_{1}}}$ with $g_{3}(\xi)=0, \forall \gamma, \gamma \leqslant \gamma_{2},\left\|g_{3}\right\| \leqslant r$ and $\left\|P_{2} T g_{3}\right\| \leqslant \sqrt[p]{\varepsilon+s}$; so there exists $\beta_{2} \in\left[\lambda_{1}+1, \omega^{\alpha_{1}}\right]$ such that $\| T\left(g_{3}\right)\left(\omega^{\alpha_{1}(N-1)} \beta_{1}^{\prime}+\omega^{\alpha_{1}(N-2)} \beta_{2} \| \geqslant \sqrt[p]{\varepsilon+s}\right.$ and we can suppose that $\beta_{2}$ is not a limit ordinal satisfying $\left\|T\left(g_{3}\right)\left(\omega^{\alpha_{1}(N-1)} \beta_{1}^{\prime}+\omega^{\beta_{1}(N-2)} \beta_{2}\right)\right\| \geqslant \sqrt[p]{\varepsilon}$. Let $\beta_{2}=\beta_{2}^{\prime}+1$. Since $T\left(g_{3}\right) \in Y^{\omega^{\alpha_{1} \omega}}$, we can find $\lambda_{2}, \lambda_{2} \in\left[1, \omega^{\alpha_{1}}\right]$ such that $\forall \gamma, \gamma \in\left[\omega^{\alpha_{1}(N-1)} \beta_{1}^{\prime}+\right.$ $\left.\omega^{\alpha_{1}(N-2)} \beta_{2}^{\prime}+\omega^{\alpha_{1}(N-3)} \lambda_{2}+1, \omega^{\alpha_{1}(N-1)} \beta_{1}^{\prime}+\omega^{\alpha_{1}(N-2)} \beta_{2}^{\prime}+1\right]$. We have $\left\|T\left(g_{3}\right)\right\| \geqslant \sqrt[p]{\varepsilon}$.

Repeating this procedure $N$ times, we can find $g^{1}=g_{1}+f_{1}, g_{1} \in Z_{0}^{\omega^{\alpha_{1}}}, f_{1} \in X$, $g_{2}, g_{3}, \ldots, g_{N} \in Z_{0}^{\omega^{\alpha_{1}}}$, ordinals $\gamma_{1}<\gamma_{2}<\cdots<\gamma_{N-1}<\omega^{\alpha_{1}}$, non-empty intervals $\Delta_{1}=\left[1, \omega^{\alpha_{1} N}\right], \Delta_{2}=\left[\omega^{\alpha_{1}(N-1)} \beta_{1}^{\prime}+\omega^{\alpha_{1}\left(N_{1}\right)} \lambda_{1}+1, \omega^{\alpha_{1}(N-1)} \beta_{1}^{\prime}+1\right], \ldots, \Delta_{n}$, such that

(1) $\Delta_{1} \supset \Delta_{2} \supset \cdots \supset \Delta_{N}$;

(2) $\left\|g^{1}\right\| \leqslant r$ and $\left\|g_{i}\right\| \leqslant r, i=1,2, \ldots, N$;

(3) $\left\|g_{i}(\gamma)\right\| \leqslant(\varepsilon / N), \forall \gamma, \gamma \in\left[\gamma_{i+1}, \omega^{\alpha_{1}}\right], i=1,2, \ldots, N-1$;

(4) $g_{i}(\gamma)=0, \forall \gamma, \gamma \in\left[1, \gamma_{i+1}\right], i=2,3, \ldots, N$;

(5) $T g^{1}(\xi)=y_{1}, \forall \xi \in \Delta_{1},\left\|T g_{1}(\gamma)\right\| \geqslant \sqrt[p]{\varepsilon}, \forall i=2, \ldots, N$, and $\forall \gamma, \gamma \in \Delta_{i}$; 
(6) we take $\gamma \in \bigcap_{i=1}^{n} \Delta_{i}$, so, by the initial remark of this proof, there exists $c_{i} \in \mathbb{R}$, with $\left|c_{i}\right|=1, i=1,2, \ldots, N$, such that $\left\|c_{1} T\left(g_{1}\right)+\cdots+c_{N} T\left(g_{N}\right)\right\| \geqslant \sqrt[p]{1+(N-1) \delta \varepsilon} ;$

(7) $\left\|c_{1} g_{1}+\cdots+c_{N} g_{N}\right\|=\max \left\{\left\|f_{1}\right\|,\left\|c_{1} g_{1}+\cdots+c_{N} g_{N}\right\|\right\} \leqslant r+\varepsilon$.

From (6) and (7) we conclude that $\sqrt[p]{1+(N-1) \delta \varepsilon} \leqslant\|T\|(r+\varepsilon)$, which is absurd because of the choices of $\varepsilon$ and $N$.

Corollary 5.5. Let $X$ and $F$ be Banach spaces and $\alpha$ an infinite denumerable ordinal, $F$ uniformly convex. If $F^{\alpha} \oplus X \rightarrow F^{\alpha^{\omega}}$, then there exists $n, m<\omega$, such that $F^{n} \oplus X^{m} \rightarrow$ $F^{\alpha^{\omega}}$.

Proof. Let $\alpha_{0}=\min \left\{\xi: \exists m, m<\omega, X^{m} \oplus F^{\xi} \rightarrow F^{\alpha}\right\}$. So $\alpha_{0} \leqslant \alpha$, and if we suppose that $\alpha_{0} \geqslant \omega$, then there exists $n_{0}<\omega$ such that (a) $X^{n_{0}} \oplus F^{\alpha_{0}} \rightarrow F^{\alpha}$, and (b) $\forall \beta, \beta<\alpha_{0}, F^{\beta} \oplus X^{n_{0}+1} \not \nrightarrow F^{\alpha_{0}}$, otherwise there exists $\beta, \beta<\alpha_{0}$, such that $X^{2 n_{0}+1} \oplus F^{\beta} \rightarrow X^{n_{0}+1} \oplus F^{\beta} \oplus X^{n_{0}} \rightarrow F^{\alpha_{0}} \oplus X^{n_{0}} \rightarrow F^{\alpha}$, which is absurd because of the choice of $\alpha_{0}$. Therefore, from Proposition 5.4, it follows that $F^{\alpha_{0}} \oplus X^{n_{0}+1} \not \nrightarrow F^{\alpha_{0}^{\omega}}$.

However, from (a) and our hypothesis it follows that $F^{\alpha_{0}} \oplus X^{n_{0}+1}=F^{\alpha_{0}} \oplus X \oplus X^{n_{0}} \rightarrow$ $X \oplus F^{\alpha} \rightarrow F^{\alpha^{w}} \rightarrow F^{\alpha_{0}^{\omega}}$, which is absurd. Consequently, $\alpha_{0}<\omega$, and again from (a) we have $X^{n_{0}} \oplus F^{\alpha_{0}} \oplus X \rightarrow F^{\alpha} \oplus X \rightarrow F^{\alpha^{\omega}}$.

Proof of Theorem 5.1. If $\xi^{\omega} \leqslant \eta$, then writing $G=F^{*}$, we have

$$
X \oplus G^{\xi} \rightarrow X^{\xi} \oplus G^{\xi} \sim(X \oplus G)^{\xi} \rightarrow(X \oplus G)^{\eta} \rightarrow G^{\eta} \rightarrow G^{\xi^{\omega}} .
$$

From Corollary 5.5 it follows that there exists $n, m<\omega$, such that $G^{n} \oplus X^{m} \rightarrow$ $G^{\xi^{\omega}} \rightarrow G^{\omega}$. Now, bearing in mind that every uniformly convex Banach space is reflexive, see Proposition 1.e.3 of [13], we have $F^{n+2} \hookrightarrow\left(G^{\omega}\right)^{*} \hookrightarrow F^{n} \oplus\left(X^{*}\right)^{m}$, that is there exists $T: F^{n+2} \rightarrow F^{n} \oplus\left(X^{*}\right)^{m}$ an isomorphism onto the image; $T\left(F^{n+2}\right)$ and $\left(X^{*}\right)^{m}$ being totally incomparable Banach spaces, it follows that $T\left(F^{n+2}\right) \cap\left(X^{*}\right)^{m}=V$, where $\operatorname{dim} V=p, p<\omega$ and, therefore, $T\left(F^{n+2}\right)=Z \oplus V$ for some Banach space $Z$. Noticing that $Z \subset\left(X^{*}\right)^{m} \oplus F^{n}, Z \cap\left(X^{*}\right)^{m}=\{0\}$ and $Z$ and $X^{*}$ are totally incomparable, we have from Lemma 1.1 in $[\mathbf{2 0}]$ that $Z \hookrightarrow F^{n}$, and so $T\left(F^{n+2}\right)=Z \oplus V \hookrightarrow F^{n} \oplus \mathbb{R}^{p} \hookrightarrow F^{n+1}$, which is absurd.

Let $p$ be a real number, $p>2$. It follows from the main result of $[\mathbf{6}]$ that there exists finite-dimensional uniformly convex Banach spaces $X_{i}, i=1,2, \ldots$, such that if $F$ is the $p$ sum of these spaces, then $F^{n+1} \nrightarrow F^{n}, \forall n, 1 \leqslant n<\omega$.

It is well known that every infinite-dimensional subspace of $F$ contains a subspace isomorphic to $\ell_{p}$ and that $\ell_{p} \hookrightarrow C(I)^{*}$ (see [2, p. 207]). So $F$ and $C(I)^{*}$ are totally incomparable and we have the following corollary.

Corollary 5.6. $\left(C(I) \oplus F^{*}\right)^{\xi} \sim\left(C(I) \oplus F^{*}\right)^{\eta}$, with $\omega \leqslant \xi \leqslant \eta<\omega_{1}$, if and only if $\eta<\xi^{\omega}$.

Theorem 5.7. Let $\xi, \omega \leqslant \xi<\omega_{1}$, and let $X$ be a Banach space. If $X^{\xi} \rightarrow \mathbb{R}^{\xi^{\omega}}$, then $X \rightarrow \mathbb{R}^{\omega}$. 
Proof. Let $\xi_{0}=\min \left\{\eta \geqslant \omega: X^{\eta} \rightarrow \mathbb{R}^{\eta^{\omega}}\right\}$. If $\xi_{0}>\omega$, then $X^{\beta} \not \nrightarrow \mathbb{R}^{\xi_{0}}, \forall \beta, \omega \leqslant \beta<\xi_{0}$, otherwise there exists $\beta, \omega \leqslant \beta<\xi_{0}$, such that $X^{\beta} \rightarrow \mathbb{R}^{\xi_{0}}$.

If $\beta^{\omega}<\xi_{0}^{\omega}$, it follows from Theorem 1 of $[\mathbf{1}]$ that $X^{\beta} \rightarrow \mathbb{R}^{\xi_{0}} \rightarrow \mathbb{R}^{\beta^{\omega}}$, which is absurd because of the choice of $\xi_{0}$.

If $\beta^{\omega}=\xi_{0}^{\omega}$, again from Theorem 1 of $[\mathbf{1}]$ we have $X^{\beta} \sim X^{\xi_{0}} \rightarrow \mathbb{R}^{\xi_{0}^{\omega}}=\mathbb{R}^{\beta^{\omega}}$, which, again, is absurd.

From Proposition 5.4 it follows that $X^{\xi_{0}} \nrightarrow \nrightarrow \mathbb{R}^{\xi_{0}^{\omega}}$, which is a contradiction.

Consequently, $\xi_{0}=\omega$ and, therefore, $X^{\omega} \rightarrow \mathbb{R}^{\omega^{\omega}}$, and again from Proposition 5.4 it follows that there exists $n<\omega$ such that $X^{\omega} \rightarrow \mathbb{R}^{\omega}$, and from Theorem 2 of $[\mathbf{1 7}]$ we have $X \rightarrow \mathbb{R}^{\omega}$.

Corollary 5.8. $\ell_{\infty}(\mathbb{N})^{\xi} \sim \ell_{\infty}(\mathbb{N})^{\eta}$, with $\omega \leqslant \xi \leqslant \eta<\omega_{1}$, if and only if $\eta<\xi^{\omega}$.

Proof. If $\xi^{\omega} \leqslant \eta$, then $\ell_{\infty}(\mathbb{N})^{\xi} \rightarrow \ell_{\infty}(\mathbb{N})^{\eta} \rightarrow \mathbb{R}^{\eta} \rightarrow \mathbb{R}^{\xi^{\omega}}$, so, by the above theorem, $\ell_{\infty}(\mathbb{N}) \rightarrow \mathbb{R}^{\omega}$, which is absurd because $\mathbb{R}^{\omega}$ is not reflexive, see the theorem on p. 304 in $[\mathbf{1 8}]$.

Our results suggest the following.

Definition 5.9. We say that the Banach space $X$ is $\omega_{1}$ cancellable if $X^{\xi} \sim X^{\eta}$ with $\xi \leqslant \eta<\omega_{1}$ implies $\eta<\xi^{w}$.

Question 5.10. Give an isomorphic characterization of the separable $\omega_{1}$ cancellable Banach spaces.

\section{Remarks and questions about the Banach spaces $\mathbb{R}^{\xi}, \omega \leqslant \xi<\omega_{1}$}

Corollary 6.1 follows from Corollary 5.5, so we put Question 6.2.

Corollary 6.1. Let $\alpha$ be an infinite denumerable ordinal and $X$ a Banach space. If $\mathbb{R}^{\alpha} \oplus X \rightarrow \mathbb{R}^{\alpha^{\omega}}$, then there exists $m, m<\omega$, such that $X^{m} \rightarrow \mathbb{R}^{\alpha^{\omega}}$.

Question 6.2. If $X$ is a Banach space such that there exists $m<\omega$ and $\alpha, \omega \leqslant \alpha<\omega_{1}$ with $X^{m} \rightarrow \mathbb{R}^{\alpha^{\omega}}$, then is it true that $X \rightarrow \mathbb{R}^{\alpha^{\omega}}$ ?

Definition 6.3. Let $\alpha$ be an infinite ordinal. We say that the Banach space $X$ has the $S Q(\alpha)$ property, if, for every $\gamma, \omega \leqslant \gamma \leqslant \alpha$, such that $X \rightarrow \mathbb{R}^{\gamma}$, we have $X \rightarrow \mathbb{R}^{\gamma} \oplus X$.

Remark 6.4. It is clear that if the Banach space $X$ satisfies $X \rightarrow X^{2}$, then $X$ has the $S Q(\alpha)$ property $\forall \alpha, \alpha \geqslant \omega$, and, if $G=F^{*}$, where $F$ is the space of Figiel, then $G$ has the $S Q(\alpha)$ property $\forall \alpha, \alpha \geqslant \omega$, but $G \nrightarrow \nrightarrow G^{2}$.

Question 6.5. Give a Banach space that does not have the $S Q(\alpha)$ property for some $\alpha, \omega \leqslant \alpha<\omega_{1}$.

Theorem 6.6. Let $\xi$ and $\eta$ be infinite denumerable ordinals and let $X$ be a Banach space having the $S Q(\xi)$ property. If $\mathbb{R}^{\xi} \oplus X \rightarrow \mathbb{R}^{\eta}$, then either $X \rightarrow \mathbb{R}^{\eta}$ or $\eta<\xi^{\omega}$. 
Proof. We will prove by transfinite induction on $\eta$ that: $\forall \xi, \omega \leqslant \xi<\omega_{1}$, and for every Banach space $X$ having the $S Q(\xi)$ property, with $\mathbb{R}^{\xi} \oplus X \rightarrow \mathbb{R}^{\eta}$, then either $X \rightarrow \mathbb{R}^{\eta}$ or $\eta<\xi^{\omega}$.

If $\eta=\omega$, then, since $\xi \geqslant \omega$, we have $\eta<\xi^{\omega}$.

Now, we suppose that this result is true for every ordinal $\varphi, \omega \leqslant \varphi<\theta$, and we consider $\mathbb{R}^{\xi} \oplus X \rightarrow \mathbb{R}^{\theta}$, with $X$ having the $S Q(\xi)$ property. If $\xi^{\omega} \leqslant \theta$, then $\mathbb{R}^{\xi} \oplus X \rightarrow \mathbb{R}^{\xi^{\omega}}$. Let $\gamma=\min \left\{\beta: \mathbb{R}^{\beta} \rightarrow \mathbb{R}^{\xi}\right\}$, so $\omega \leqslant \gamma \leqslant \xi, \gamma<\gamma^{\omega} \leqslant \xi^{\omega} \leqslant \theta$ and, since $\mathbb{R}^{\gamma} \rightarrow \mathbb{R}^{\xi}$, it follows that $\mathbb{R}^{\gamma} \oplus X \rightarrow \mathbb{R}^{\xi} \oplus X \rightarrow \mathbb{R}^{\xi^{\omega}} \rightarrow \mathbb{R}^{\gamma^{\omega}}$, and by Proposition 5.4 we have

$$
X \oplus \mathbb{R}^{\gamma_{1}} \rightarrow \mathbb{R}^{\gamma} \text { for some } \omega \leqslant \gamma_{1}<\gamma .
$$

By the choice of $\gamma$ we conclude that $\mathbb{R}^{\gamma_{1}} \not \nrightarrow \mathbb{R} \mathbb{R}^{\gamma}$, so from $[\mathbf{1}]$ it follows that $\gamma_{1}^{\omega} \leqslant \gamma$, and, since $X$ has the $S Q\left(\gamma_{1}\right)$ property, using the hypothesis of induction at (6.1) we have that $X \rightarrow \mathbb{R}^{\gamma} \rightarrow \mathbb{R}^{\xi}$, and, since $X$ has the $S Q(\xi)$ property, we conclude that $X \rightarrow \mathbb{R}^{\xi} \oplus X \rightarrow \mathbb{R}^{\theta}$.

Question 6.7. Let $X$ and $Y$ be separable Banach spaces and $\xi, \omega^{\omega} \leqslant \xi<\omega_{1}$. If $X \oplus Y \rightarrow \mathbb{R}^{\xi}$, then is it true that either $X \rightarrow \mathbb{R}^{\xi}$ or $Y \rightarrow \mathbb{R}^{\xi}$ ?

Since $\mathbb{R} \oplus \mathbb{R}^{\xi} \sim \mathbb{R}^{\xi}, \forall \xi, \xi \geqslant \omega, \mathbb{R}^{\xi}$ is isomorphic to each of its closed hyperplanes. The following lemma gives a positive answer to the above question in the case in which either $X$ or $Y$ is a finite-dimensional space.

Lemma 6.8. Let $X$ and $H$ be Banach spaces such that $H$ is isomorphic to each of its closed hyperplanes. If $\mathbb{R} \oplus X \rightarrow H$, then $X \rightarrow H$.

Proof. Let $T: \mathbb{R} \oplus X \rightarrow H$ be a surjective bounded linear operator. If $T(1,0)=0$, then $\left.T\right|_{X}: X \rightarrow H$ is surjective. If $T(1,0)=h_{1} \neq 0$, then writing $H=\left[h_{1}\right] \oplus H_{1}$ for some closed hyperplane $H_{1}$ of $H$ and indicating by $P$ the canonical projection from $H$ onto $H_{1}$, we have $P T(1,0)=0$, therefore $\left.P T\right|_{H_{1}}: X \rightarrow H_{1}$ is onto $H_{1}$, and, from the hypothesis $H \sim H_{1}$, we have $X \rightarrow H$.

Question 6.9. If $X$ and $Y$ are Banach spaces such that $\mathbb{R} \oplus X \rightarrow H$, and $H$ is of infinite dimension, then is it true that $X \rightarrow H$ ?

Corollary 6.10. Let $\alpha$ be an infinite denumerable ordinal and let $X$ be a Banach space. If $X^{\alpha} \rightarrow \mathbb{R}^{\alpha^{\omega}}$, then there exists $n, m<\omega$, such that $\left(\hat{\otimes}_{m} X\right)^{n} \rightarrow \mathbb{R}^{\alpha^{\omega}}$.

Proof. It suffices to take $\alpha_{0}=\min \left\{\xi: \exists m, m<\omega,\left(\hat{\otimes}_{m} X\right)^{\xi} \rightarrow \mathbb{R}^{\alpha}\right\}$ and to proceed as in the Lemma 3.4 using Proposition 5.4.

Question 6.11. If $X$ is a Banach space such that there exists $n, n<\omega$ and $\alpha$, $\omega \leqslant \alpha<\omega_{1}$ with $\hat{\otimes}_{n} X \rightarrow \mathbb{R}^{\alpha^{\omega}}$, then is it true that $X \rightarrow \mathbb{R}^{\alpha}$ ?

Definition 6.12. Let $\alpha$ be an infinite ordinal. We say that the Banach space $X$ has the $T Q(\alpha)$ property if, for every $\gamma, \omega \leqslant \gamma \leqslant \alpha$, such that $X \rightarrow \mathbb{R}^{\gamma}$, we have $X \rightarrow X^{\gamma}$.

Remark 6.13. It is clear that if the Banach space $X$ satisfies $X \rightarrow X \hat{\otimes} X$, then $X$ has the $T Q(\alpha)$ property $\forall \alpha, \alpha \geqslant \omega$. 
Theorem 6.14. Let $\xi$ and $\eta$ be infinite denumerable ordinals and let $X$ be a Banach space having the $T Q(\xi)$ property. If $X^{\xi} \rightarrow \mathbb{R}^{\eta}$, then either $X \rightarrow \mathbb{R}^{\eta}$ or $\eta<\xi^{\omega}$.

Proof. Analogous to that for Theorem 6.8.

Question 6.15. Give a Banach space that does not have the $T Q(\alpha)$ property for some $\alpha, \omega \leqslant \alpha<\omega_{1}$.

\section{References}

1. C. Bessaga And A. Pelczynski, Spaces of continuous functions, IV, Studia Math. XIX (1960), 53-61.

2. C. Bessaga And A. Pelczynski, Some aspects of the present theory of Banach spaces, in Appendix of theory of linear operators (ed. S. Banach) (North-Holland Mathematical Library, 1987).

3. N. Bourbaki, General topology, part 2 (Hermann, Paris, 1966).

4. J. Diestel And J. J. J. R. Uhl, Vector measures (American Mathematical Society, Providence, RI, 1977).

5. N. Dunford And J. T. Schwartz, Linear operators, I, General theory (Interscience, New York, 1958).

6. T. Figiel, Example of infinite dimensional reflexive Banach space non-isomorphic to its Cartesian square, Studia Math. XLII (1972), 295-306.

7. E. M. GALEGO, How to generate new Banach spaces non-isomorphic to their Cartesian squares, Bull. Acad. Pol. Sci. 47 (1999), 21-25.

8. W. Hurewicz, Zur theorie der analytischen mengen, Fund. Math. 15 (1930), 4-17.

9. T. KAPPELER, Banach spaces with condition of Mazur's, Math. Z. 191 (1986), 623-631.

10. S. V. KisLyakov, Classification of spaces of continuous functions of ordinals, Siberian Math. J. 16 (1975), 226-231.

11. D. LeunG, On Banach spaces with Mazur's property, Glasgow Math. J. 33 (1991), 51-54.

12. J. Lindenstrauss and A. Pelczynski, Contributions to the theory of the classical Banach spaces, J. Funct. Analysis 8 (1971), 225-249.

13. J. Lindenstrauss And L. TZafriri, Classical Banach spaces, II, Function spaces (Springer, New York, 1979).

14. H. P. Rosenthal, Some recent discoveries in the isomorphic theory of Banach spaces, Bull. Am. Math. Soc. 5 (1978), 803-831.

15. H. P. Rosenthal, On applications of the boundedness principle to Banach space theory according to J. Bourgain (Exp. no. 5, Pub. Math. Univ. Pierre et Marie Curie, 29 Univ. Paris VI, 1979).

16. C. SAmuel, Contributions à l'étude des espaces de Banach, PhD thesis, l'Université d'AixMarseille II (1977).

17. C. Samuel, Sur la reproductibilité des espaces $l_{p}$, Math. Scand. 45 (1979), 103-117.

18. Z. Semadeni, Banach space of continuous functions (PWN-Polish Scientific Publishers, Warzawa, 1971).

19. A. Wilansky, Mazur spaces, Internet J. Math. Sci. 4 (1981), 39-53.

20. P. WojtaszczyK, On complemented subspaces and unconditional bases in $\ell_{p}+\ell_{q}$, Studia Math. XLV (1973), 197-206. 